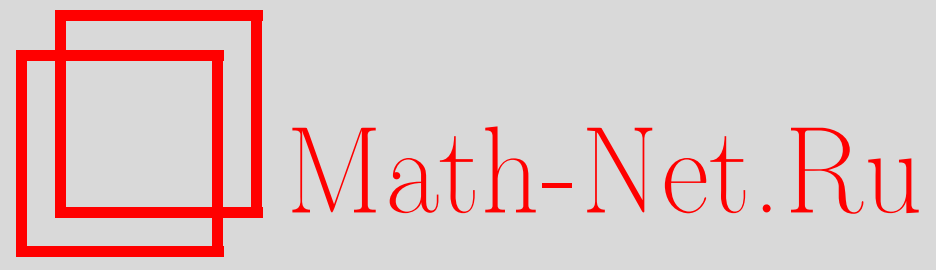

С. П. Чулков, О числе Милнора эквивариантной особенности, Матем. заметки, 2002, том 71, выпуск 6, 950-953

DOI: https://doi.org/10.4213/mzm657

Использование Общероссийского математического портала Math-Net.Ru подразумевает, что вы прочитали и согласны с пользовательским соглашением http://www. mathnet.ru/rus/agreement

Параметры загрузки:

IP : 54.162 .27 .143

26 апреля 2023 г., $17: 42: 16$

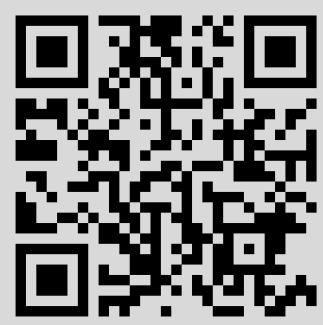




\section{О ЧИСЛЕ МИЛНОРА ЭКВИВАРИАНТНОЙ ОСОБЕННОСТИ}

\section{С. П. Чулков}

Введение. Мы доказываем нижнюю оценку на число Милнора конечнократного ростка голоморфной функции с нулевой 2-струей инвариантного относительно нетривиального представления группы $\mathbb{Z}_{p}$ ( $p$-простое). Эта оценка позволяет дать новое (отличное от приведенного в [1]) доказательство гипотезы Казаряна о ростках функций, инвариантных относительно действия компактных групп Ли.

Рассмотрим пространство $\mathbb{C}^{n}$ с нетривиальным представлением группы $\mathbb{Z}_{p}(p-$ простое число) на нем.

Теорема. Допустим, что $f:\left(\mathbb{C}^{n}, 0\right) \rightarrow(\mathbb{C}, 0)-$ инвариантный относительно представления әруппы $\mathbb{Z}_{p}$ росток голоморфной функции с изолированной критической точкой в 0 и его 2-струя $j_{0}^{2} f$ равна 0 . Тогда для числа Милнора $\mu(f)$ ростка $f$ в 0 выполнено неравенство $\mu(f) \geqslant(p-1)$.

Обозначим через $\operatorname{Diff}_{0}\left(\mathbb{C}^{n}\right)$ группу ростков дифффеоморфизмов $\left(\mathbb{C}^{n}, 0\right) \rightarrow\left(\mathbb{C}^{n}, 0\right)$.

СледствиЕ. Предположим, что $f:\left(\mathbb{C}^{n}, 0\right) \rightarrow(\mathbb{C}, 0)-$ росток голоморфной функиии $c$ изолированной критической точкой в 0 и его 2 -струя $j_{0}^{2} f$ равна 0 . Пусть $G$ - некоторая компактная группа Ли его симметрий, т.е. подгруппа $\operatorname{Diff}_{0}\left(\mathbb{C}^{n}\right)$ maкая, что $f \circ g \equiv f$ для любого $g \in G$. Тогда $G$ дискретна.

ЗАмеч АниЕ. Последнее утверждение было высказано Казаряном в качестве гипотезы (см. [2] и его же приложение к русскому переводу книги [3]) и было доказано Васильевым в [1].

ДоКАЗАТЕЛЬСТво СЛЕДСтвИЯ. Предположим противное. Так как голоморфное действие компактной группы Ли на комплексном гладком многообразии вблизи своей неподвижной точки голоморфино эквивалентно линейному (см., например, [4]), мы можем полагать, что наша группа $G$ является подгруппой $G L\left(\mathbb{C}^{n}\right)$. Кроме того, мы можем считать, что $G=S^{1}$, так как любая недискретная компактная группа Ли содержит такую подгруппу. Отождествив ее с единичной окружностью в $\mathbb{C}^{1}$, получаем, что для каждого простого $p$ на пространстве $\mathbb{C}^{n}$ возникает представление группы $\mathbb{Z}_{p}$ - мультипликативной группы корней $p$-ой степени из единицы. Для каждого $p$ росток $f$ инвариантен относительно этого представления, но для достаточно больших $p$ представление нетривиально. Следовательно, применив основную теорему, мы приходим к противоречию с условием изолированности $f$.

Следующая серия примеров показьвает, что теорема дает точную по порядку величины оценку. А именно при фиксированном $n$ нижняя оценка числа Милнора для представлений без тривиальых слагаемых линейна по $p$ (можно представить себе, что она могла бы расти как $p$ в какой-то степени, зависящей от $n$ ).

ПримеР 1. Рассмотрим следующие представление группы $\mathbb{Z}_{p}(p>2$-простое число) на пространстве $\mathbb{C}^{n}=\left\{z=\left(z_{1}, \ldots, z_{n}\right) \mid z_{i} \in \mathbb{C}\right\}$ при $n \geqslant 2$ :

$$
T_{g}:\left(z_{1}, z_{2}, \ldots, z_{2 l}, z_{2 l+1}, \ldots, z_{n}\right) \mapsto\left(g z_{1}, g^{p-2} z_{2}, \ldots, g^{p-2^{2 l-1}} z_{2 l}, g^{2^{2 l}} z_{2 l+1}, \ldots\right), \quad g \in \mathbb{Z}_{p}
$$


Здесь и далее мы отождествляем группу $\mathbb{Z}_{p}$ с мультипликативной группой корней $p$-й степени из единицы. Тогда функция $f_{n, p}(z)=z_{1}^{2} z_{2}+z_{2}{ }^{2} z_{3}+z_{3}{ }^{2} z_{4}+\cdots+z_{(n-1)}{ }^{2} z_{n}+z_{n}{ }^{p}$ инвариантна относительно данного представления. Число Милнора функции $f_{n, p}$ в нуле равно локальноу индексу нулевого решения следующей системы уравнений

$$
\frac{\partial f_{n, p}}{\partial z_{1}}(z)=\frac{\partial f_{n, p}}{\partial z_{2}}(z)=\cdots=\frac{\partial f_{n, p}}{\partial z_{n}}(z)=0 .
$$

В нашем случае получаем систему

$$
\left\{\begin{aligned}
2 z_{1} z_{2} & =0, \\
z_{1}^{2}+2 z_{2} z_{3} & =0, \\
z_{2}^{2}+2 z_{3} z_{4} & =0, \\
\ldots \ldots \ldots \ldots \cdots \cdots & \ldots \\
z_{n-1}^{2}+p z_{n}^{p-1} & =0 .
\end{aligned}\right.
$$

Индукцией по $n$ легко показать, что нулевое решение системы $(* n)$ изолировано, следовательно, проводимые вычисления корректны. Явный вид $(* n)$ дает рекурентное соотношение на индекс системы и, следовательно, на числа Милнора ростков $f_{n, p}$, при различных $n$. А именно

$$
\mu\left(f_{n, p}\right)=\mu\left(f_{n-1, p}\right)+2 \mu\left(f_{n-2, p}\right) .
$$

Откуда находим, что $\mu\left(f_{n, p}\right)=\left(2^{n} p+(-1)^{n+1}(p-3)\right) / 3$.

Заметим, что в этой серии примеров начало координат является единственной неподвижной точкой.

Следующая серия примеров показывает, что аналогичная (по порядку величины) оценка для $\mathbb{Z}_{m}$, где $m$ не обязательно простое, неверна.

ПримеР 2. Рассмотрим следующее представление группы $\mathbb{Z}_{m}$ (мы отождествляем $\mathbb{Z}_{m}$ с мультипликативной группой корней $m$-й степени из единицы) при $m=p_{1} p_{2}\left(p_{1}+p_{2}\right)$, где $p_{1}$ и $p_{2}-$ различные простые числа, на пространстве $\mathbb{C}^{3}=\left\{z=\left(z_{1}, z_{2}, z_{3}\right) \mid z_{i} \in \mathbb{C}\right\}$ :

$$
T_{g}:\left(z_{1}, z_{2}, z_{3}\right) \mapsto\left(g^{p_{2}} z_{1}, g^{p_{1}} z_{2}, g^{m-p_{1}-p_{2}} z_{3}\right), \quad g \in \mathbb{Z}_{m}
$$

Тогда функция $f(z)=z_{1} p_{1}\left(p_{1}+p_{2}\right)+z_{2} p_{2}\left(p_{1}+p_{2}\right)+z_{3} p_{1} p_{2}+z_{1} z_{2} z_{3}$ инвариантна относительно данного представления и имеет изолированную критическую точку в начале координат. По формуле Кушниренко для числа Милнора (см., например, [5]) получаем, $\mu(f)=p_{1} p_{2}+\left(p_{1}+p_{2}\right)^{2}-1$.

Заметим, что некоторые точки $\mathbb{C}^{3}$ имеют орбиты максимально возможной длины $-m$, т.е. данное представление не пропускается через какую-либо подгруппу.

Доказательства. Сделав подходящую замену координат, можно считать, что представление группы $\mathbb{Z}_{p}$ имеет следующий вид:

$$
T_{g}:\left(z_{1}, \ldots, z_{n}\right) \mapsto\left(g^{a_{1}} z_{1}, \ldots, g^{a_{n}} z_{n}\right),
$$

где $T_{g}$ - линейный оператор, соответствующий элементу $g \in \mathbb{Z}_{p}, \mathrm{a} a_{1}, \ldots, a_{n}\left(0 \leqslant a_{i}<p\right)$ - фиксированные целые числа. Не ограничивая общности будем полагать, что если среди чисел $a_{1}, \ldots, a_{n}$ есть равные нулю, то это первые $k$. То есть $L=\left\{z \in \mathbb{C}^{n} \mid z=\left(z_{1}, \ldots, z_{k}, 0, \ldots, 0\right), z_{i} \in \mathbb{C}\right\}$ есть подпространство неподвижных точек относительно данного представления группы $\mathbb{Z}_{p}$. Обозначим через $L_{g}$ подпространство неподвижных точек оператора $T_{g}$, а через $\mu\left(\left.f\right|_{L_{g}}\right)$ число Милнора ограничения ростка $f$ на подпространство $L_{g}$ в точке 0 (ограничение $\left.f\right|_{L_{g}}$ имеет конечнократную (изолированную) особенность в $0 \in L_{g}$, см., например, [6]). Если $L_{g}$ есть только точка 0, считаем, что $\mu\left(\left.f\right|_{L_{g}}\right)=1$. Так как $p$ простое, то для всех $g \in \mathbb{Z}_{p}$, отличных от 1, пространство $L_{g}=L$, а для 1 пространство $L_{1}$ совпадает со всем $\mathbb{C}^{n}$.

ЛЕмма 1. Пусть $f:\left(\mathbb{C}^{n}, 0\right) \rightarrow(\mathbb{C}, 0)$ - росток голоморфной функиии с изолированной особой точкой в 0 и его 2 -струя $j_{0}^{2} f$ равна 0 . Предположим также, что $f$ инвариантен относительного нетривиального представления группы $\mathbb{Z}_{p}$. Тогда $\mu(f)>\mu\left(\left.f\right|_{L}\right)$. 
ДОКАЗАТЕЛЬСТВО. Если $k=0$, т.е. единственной неподвижной точкой действия группы является начало координат, то утверждение леммы, очевидно, выполнено. Предположим, что $k>0$. Пусть $I_{\nabla f}$ - идеал кольца $\mathscr{O}_{\mathbb{C}^{n}, 0}$ голоморфных ростков в $\left(\mathbb{C}^{n}, 0\right)$, порожденный множеством $\left\{\partial f / \partial z_{1}, \ldots, \partial f / \partial z_{n}\right\} ; I_{\nabla}\left(\left.f\right|_{L}\right)$ - идеал кольца $\mathscr{O}_{L, 0}$ голоморфных ростков в $(L, 0)$, порожденный множеством $\left\{\partial\left(\left.f\right|_{L}\right) / \partial z_{1}, \ldots, \partial\left(\left.f\right|_{L}\right) / \partial z_{k}\right\}$. Обозначим через $A=\mathscr{O}_{\mathbb{C}^{n}, 0} / I_{\nabla f}$ и $B=\mathscr{O}_{L, 0} /$ $I_{\nabla\left(\left.f\right|_{L}\right)}$ соответствуюшие факторпространства. Имеем $\mu(f)=\operatorname{dim}_{\mathbb{C}} A$ и $\mu\left(\left.f\right|_{L}\right)=\operatorname{dim}_{\mathbb{C}} B($ см., например, [7]). Пусть $a_{1}, \ldots, a_{l} \in \mathbb{C}\left[z_{1}, \ldots, z_{k}\right]$ - представители какого-нибудь базиса $B$. Тогда никакая нетривиальная линейная комбинации с комплексными коэффициентами многочленов $a_{1}, \ldots, a_{l}$ (рассматриваемых как элементы $\mathscr{O}_{\mathbb{C}}, 0$ ) не принадлежит идеалу $I_{\nabla f}$. Действительно, в силу эквивариантности разложение $f$ в ряд Тейлора не может содержать мономы вида

$$
a z_{j} \prod_{i=1}^{k} z_{i}^{\alpha_{i}}, \quad \text { где } j>k, \quad a \in \mathbb{C}, \quad \alpha_{i} \in \mathbb{Z}_{\geqslant 0} .
$$

Следовательно,

$$
\forall i \in\{1, \ldots, n\}:\left.\quad \frac{\partial f}{\partial z_{i}}\right|_{L}=\frac{\partial\left(\left.f\right|_{L}\right)}{\partial z_{i}}
$$

в частности,

$$
\left.\frac{\partial f}{\partial z_{i}}\right|_{L} \equiv 0 \quad \text { при } i<k .
$$

То есть для ростка $f$ операция ограничения на $L$ коммутирует с операцией взятия частной производной по любой координате, откуда и следует наше утверждение. Более того, очевидно, что никакая нетривиальная линейная комбинация многочленов $z_{k+1}, a_{1}, \ldots, a_{l}$ также не принадлежит идеалу $I_{\nabla f}$, так как $j_{0}^{2} f=0$. Следовательно, $\operatorname{dim} A>\operatorname{dim} B$.

Обозначим через $\mu_{\mathbb{Z}_{p}}(f) \in R\left(\mathbb{Z}_{p}\right)$ эквивариантное число Милнора ростка $f$ в 0, где $R\left(\mathbb{Z}_{p}\right)$ кольцо комплексных представлений группы $\mathbb{Z}_{p}$. Эквивариантное число Милнора $\mu_{\mathbb{Z}_{p}}(f)$ есть элемент $R\left(\mathbb{Z}_{p}\right)$, соответствующий группе исчезающих гомологий $f$ как векторному пространству с естественным представлением группы $\mathbb{Z}_{p}$ (см., например, [6], [8]). Элемент $V$ кольца $R(G)$ комплексных представлений конечной группы $G$ определяется своим характером $[V](g)=\left.\operatorname{tr} T_{g}\right|_{V}$ как функцией на группе $G\left(\left.\operatorname{tr} T_{g}\right|_{V}\right.$-след оператора $\left.\left.T_{g}\right|_{V}\right)$. В силу [6] значение характера, соответствующего $\mu_{\mathbb{Z}_{p}}(f)$ на элементе $g \in \mathbb{Z}_{p}$, вычисляется по формуле

$$
\left[\mu_{\mathbb{Z}_{p}}(f)\right](g)=(-1)^{1-\operatorname{dim} L_{g}} \mu\left(\left.f\right|_{L_{g}}\right) .
$$

Лемма 2. Не существует инвариантного относительно нетривиального представления группы $\mathbb{Z}_{p}$ конечнократного ростка голоморфной функчии $f:\left(\mathbb{C}^{n}, 0\right) \rightarrow(\mathbb{C}, 0) c$ нулевой 2-струей $j_{0}^{2} f=0$, эквивариантное число Милнора $\mu_{\mathbb{Z}_{p}}(f)$ которого является прямой суммой нескольких тривиальных одномерных представлений.

ДокАЗАТЕЛЬСтво. Предположим противное. Тогда $\mu(f)=\left[\mu_{\mathbb{Z}_{p}}(f)\right](1)=\left[\mu_{\mathbb{Z}_{p}}(f)\right](g)=$ $(-1)^{n-k} \mu\left(\left.f\right|_{L}\right)$ для любого нетривиального элемента $g \in \mathbb{Z}_{p}$. Следовательно, $(n-k)$ четно и $\mu(f)=\mu\left(\left.f\right|_{L}\right)$, что невозможно в силу леммы 1 .

Рассмотрим стандартное представление группы $\mathbb{Z}_{p}$ на пространстве $\mathbb{C}^{p}=\left\{\left(z_{1}, \ldots, z_{p}\right) \mid\right.$ $\left.z_{i} \in \mathbb{C}\right\}$ циклическими перестановками координат. Обозначим через $W \in R\left(\mathbb{Z}_{p}\right)$ класс представления, полученного ограничением указанного стандартного представления на гиперплоскость $z_{1}+\cdots+z_{p}=0$.

ЛЕмма 3. Пусть $f:\left(\mathbb{C}^{n}, 0\right) \rightarrow(\mathbb{C}, 0)$ - конечнократный росток голоморфной функции, инвариантный относительно нетривиального представления группь $\mathbb{Z}_{p}$. Тогда его әквивариантное число Милнора $\mu_{\mathbb{Z}_{p}}(f)$ представимо в виде

$$
\mu_{\mathbb{Z}_{p}}(f)=a \widetilde{\mathbb{C}}+b W
$$

где $a, b \in \mathbb{Z}_{\geqslant 0}$, а через $\widetilde{\mathbb{C}}$ обозначен класс тривиального одномерного представления в $R\left(\mathbb{Z}_{p}\right)$. 
ДокаЗАТЕльСтво. Обозначим через $V_{\varepsilon} \subset \mathbb{C}^{n}$ слой Милнора ростка $f$ в 0 . Так как росток $f$ инвариантен относительно представления группы $\mathbb{Z}_{p}$, на $V_{\varepsilon}$ возникает естественное действие группы. Обозначим через $\chi_{\mathbb{Z}_{p}}\left(V_{\varepsilon}\right) \in R\left(\mathbb{Z}_{p}\right)$ эквивариантную эйлерову характеристику $V_{\varepsilon}$ (см., например, [6], [8]). В силу [9] существует конечная триангуляция фактора $V_{\varepsilon} / \mathbb{Z}_{p}$, следовательн, существует конечное клеточное разбиение $V_{\varepsilon}$, согласованное с действием группы. Это означает, что каждая клетка разбиения под действием элемента группы либо переходит в другую клетку, либо соответствующее преобразование на ней совпадает с тождественньм. Фиксируем разбиение $V_{\varepsilon}$, согласованное с действием группы. В этом случае на векторных пространствах клеточных цепей $C_{i}\left(V_{\varepsilon}, \mathbb{C}\right)$ возникает естественное представление группы $\mathbb{Z}_{p}$. Обозначим через $\widetilde{C}_{i}\left(V_{\varepsilon}\right) \in R\left(\mathbb{Z}_{p}\right)$

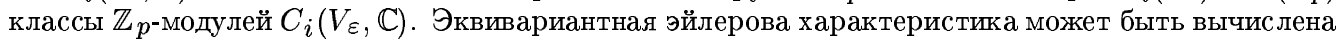
по формуле $\chi_{\mathbb{Z}_{p}}\left(V_{\varepsilon}\right)=\sum_{i=0}^{2 n}(-1)^{i} \widetilde{C}_{i}\left(V_{\varepsilon}\right)$. Но так как $p$ простое, $\widetilde{C}_{i}\left(V_{\varepsilon}\right)$, очевидно, представляются в виде $(*)$. Следовательн, эквивариантная эйлерова характеристика $\chi_{\mathbb{Z}_{p}}\left(V_{\varepsilon}\right)$ также представляется в виде $(*)$, но уже с целыми коэфффициентами. Теперь, воспользовавшись соотношением $\chi_{\mathbb{Z}_{p}}\left(V_{\varepsilon}\right)=\widetilde{\mathbb{C}}+(-1)^{n-1} \mu_{\mathbb{Z}_{p}}(f)$ и определением эквивариантного числа Милнора $\mu_{\mathbb{Z}_{p}}(f)$ (в соответствии с которым $\mu_{\mathbb{Z}_{p}}(f)$ является "настоящим", а не виртуальным представлением группы $\left.\mathbb{Z}_{p}\right)$, легко получить требуемое утверждение.

Утверждение теоремы теперь является простым следствием лемм 2 и 3 , так как лемма 2 гарантирует, что коэффициент $b$ в представлении (*) отличен от нуля и, следовательно, число Милнора

$$
\mu(f)=\left[\mu_{\mathbb{Z}_{p}}(f)\right](1)=a+b \operatorname{dim} W \geqslant p-1 .
$$

Автор благодарен С.М. Гусейн-Заде за постановку задачи и полезные обсуждения.

\section{СПИСОК ЦИТИРОВАННОЙ ЛИТЕРАТУРЫ}

1. Васильев В. А. // Функцион. анализ и его прилож. 1999. Т. 33. № 3. С. 73-75. 2. Казарян М. Э. // УМН. 1995. Т. 50. № 4. С. 45-70. 3. Васильев В. А. Лагранжевы и лежандровы характеристические классы. М.: Изд-во МЦНМО, 1999. 4. Кушниренко А. Г. // Функцион. анализ и его прилож. 1967. Т. 1. № 1. С. 103-104. 5. Кушниренко А. Г. // Функцион. анализ и его прилож. 1967. Т. 9. № 1. С. 74-75. 6. Wall С. Т. С. // Bull. London Math. Soc. 1980. V. 12. №3. Р. 169-175. 7. Арнольд В.И., Варченко А.Н., Гусейн-Заде С. М. Особенности дифференцируемых отображений. Т. 1. М.: Наука, 1982. 8. А рнольд В. И., Варченко А. Н., Гусейн-Заде С. М. Особенности дифференцируемых отображений. Т. 2. М.: Наука, 1984. 9. Yang C. T. // Bull. Amer. Math. Soc. 1963. V. 69. P. 405-408. 